\title{
Cyclotron Harmonic Waves in Warm Plasmas ${ }^{1}$
}

\author{
F. W. Crawford
}

\author{
Institute for Plasma Research, Stanford University, Stanford, Calif.
}

(Received January 11, 1965)

\begin{abstract}
It has been predicted theoretically that cyclotron harmonic waves should propagate in warm plasma confined by a magnetic field, and show cutoff and resonance behavior associated with harmonics of the cyclotron frequency and the upper hybrid resonance frequency. In this paper, a simplified analysis based on the quasi-static approximation is presented and used to establish the relevant dispersion relations. Computer solutions of these are presented showing the influence of the magnetic field and discharge parameters on the propagation. A number of recent experimental results can be explained in terms of this type of wave motion and serve to establish its validity. This work is discussed briefly. The paper discusses some of the implications of this mode in laboratory and ionospheric plasma physics.
\end{abstract}

\section{Introduction}

Wherever possible in theoretical plasma wave propagation studies the analysis is simplified by neglecting thermal motions of the charged particles. This allows the plasma to be treated as a medium possessing a frequency-dependent, effective permittivity, which may be a tensor quantity if a static magnetic field is present. This approach is familiar from the literature of ionospheric propagation and may easily be extended to include the effects of collisions [Ratcliffe, 1959; Budden, 1961].

It is recognized that the possible excitation of waves whose propagation depends on the existence of nonzero thermal velocities is ignored in the cold plasma approximation. To study these, an approach based on the Boltzmann equation is required. This may take two directions. The first possibility, the hydrodynamic approach, is to work with moments of the Boltzmann equation, and leads directly to a description of the plasma behavior in terms of macroscopic quantities such as charge density, current, and electron pressure. Analyses of this nature have led to the prediction of acoustic waves: At high frequencies these have the well-known plane-wave dispersion relation

$$
\omega^{2} \approx \omega_{p}^{2}+k^{2}\left(\frac{3 \kappa T_{e}}{m}\right),
$$

where propagation is as $\exp i(\omega t-\vec{k} \cdot \vec{r}), \omega_{p}$ is the electron plasma frequency, $\kappa$ is Boltzmann's constant, $T_{e}$ is the electron temperature, and $m$ is the electronic mass. Although (1) has never been verified experimentally, there is very strong evidence from scattering experiments carried out in the laboratory with inhomogeneous cylindrical plasmas, and described accurately by the hydrodynamic theory [Crawford, 1963, 1964a; Nickel et al., 1963; Parker et al., 1964] that the expression is a valid approximation. We shall take up this point again shortly.

At low frequencies, electrostatic sound waves are predicted with phase velocity independent of charge density and frequency. For a plane wave, the dispersion relation is,

$$
\omega \approx k\left(\frac{\kappa T_{e}}{M}\right)^{1 / 2}
$$

${ }^{1}$ This work was supported by the National Science Foundation. 
where $M$ is the ion mass. These waves have been observed in laboratory discharges [Crawford, 1961; Little, 1962].

The second method of employing the Boltzmann equation is to study individual particle trajectories, and then to integrate over velocity space to obtain macroscopic quantities. It was first pointed out by Landau [1946] that this procedure shows (1) to be incorrect. An additional damping term is required, due to trapped particles traveling with the wave [Bohm and Gross, 1949], which cannot be predicted by hydrodynamic theory. This so-called "Landau damping" becomes very strong at wavelengths comparable to a Debye length, and has been studied quantitatively recently in the laboratory [Malmberg and Wharton, 1964].

Since the single particle theory can be demonstrated to contribute damping terms to the acoustic modes predicted by hydrodynamic theory, a question now arises: does it also predict the existence of new types of propagating waves? The answer is that it does. Cyclotron harmonic waves can propagate in warm plasma immersed in a magnetic field. The objects of this paper are to demonstrate physically how these waves can occur; to give computed dispersion curves for them, and to indicate the nature of the very recent experimental evidence confirming their existence.

\section{Theory}

\subsection{Single Particle Trajectory}

First consider the dynamics of a single electron moving under the influence of a static magnetic field and a transverse rf electric field, $E_{1 x}$, given by $E_{x} \exp i\left(\omega t-k_{x} x-k_{z} z\right)$. We have, for the unperturbed trajectory of figure 1 ,

$$
\begin{aligned}
& v_{R}=v_{R}^{\prime} \quad v_{z}=v_{z}^{\prime} \\
& \phi^{\prime}=\phi+\omega_{c}\left(t^{\prime}-t\right),
\end{aligned}
$$

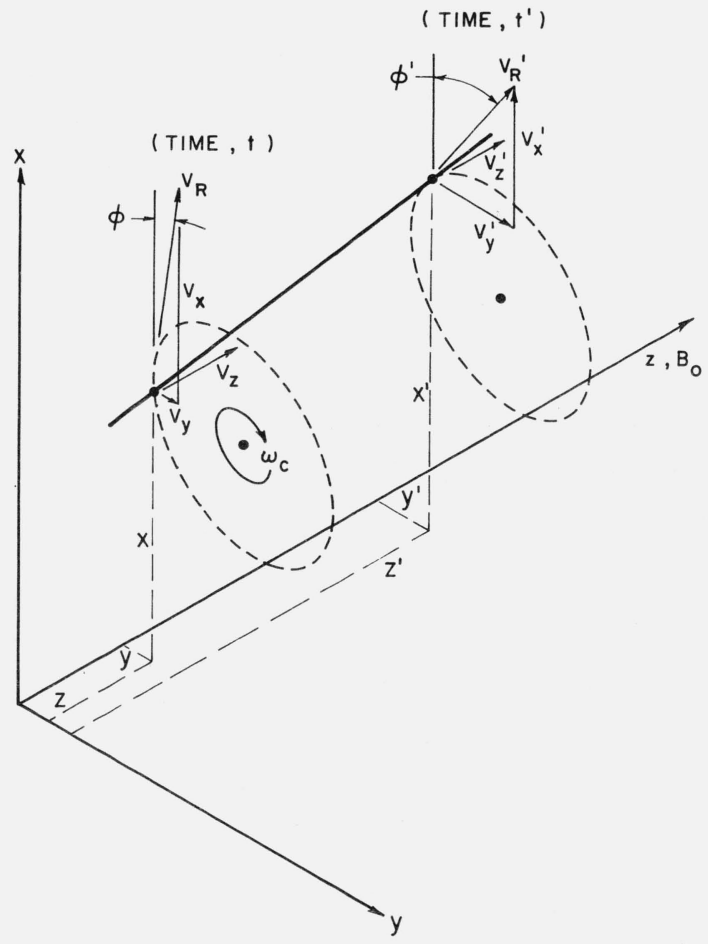

FiguRE 1. Single particle trajectory (the unperturbed motion is along a helical path, the projection of which in the $\mathrm{x}-\mathrm{y}$ plane is a circle). 
where $\omega_{c}$ is the electron cyclotron frequency. Some simple integration and trigonometric manipulation, leads to

$$
\begin{aligned}
z^{\prime} & =z+v_{z}\left(t^{\prime}-t\right) \\
x^{\prime} & =x+\left(\frac{v_{R}}{\omega_{c}}\right)\left[\sin \left\{\omega_{c}\left(t^{\prime}-t\right)+\phi\right\}-\sin \phi\right] \\
& =x+\left(\frac{2 v_{R}}{\omega_{c}}\right) \sin \left[\frac{\omega_{c}}{2}\left(t^{\prime}-t\right)\right] \cos \left[\frac{\omega_{c}}{2}\left(t^{\prime}-t\right)+\phi\right] .
\end{aligned}
$$

For the $x$-component of the perturbed trajectory, $x_{1}^{\prime}$, we have the equation of motion,

$$
\ddot{x}_{1}^{\prime}+\omega_{c}^{2} x_{1}^{\prime}=\eta E_{x} \exp i\left(\omega t^{\prime}-k_{x} x^{\prime}-k_{z} z^{\prime}\right),
$$

where $\eta$ is the electronic charge-to-mass ratio.

For small perturbations, the argument of the exponential may be replaced by its unperturbed value to obtain $x_{1}^{\prime}$ correct to first order. This yields,

$$
\begin{aligned}
\ddot{x}_{1}^{\prime}+\omega_{c}^{2} x_{1}^{\prime} & =A \exp i\left[\left(\omega-k_{z} v_{z}\right)\left(t^{\prime}-t\right)-\left(\frac{k_{x} v_{R}}{\omega_{c}}\right) \sin \left\{\omega_{c}\left(t^{\prime}-t\right)+\phi\right\}\right] \\
A & \equiv \eta E_{x} \exp i\left[\omega t-k_{x}\left(x-\left(\frac{v_{R}}{\omega_{c}}\right) \sin \phi\right)-k_{z} z\right]
\end{aligned}
$$

where we note that $\left(v_{R} / \omega_{c}\right)$ is the Larmor radius of the particle motion.

A well-known Bessel function identity may now be introduced [Bateman Manuscript Project, 1953]

$$
\exp \left[-i\left(\frac{k_{x} v_{R}}{\omega_{c}}\right) \sin \left\{\omega_{c}\left(t^{\prime}-t\right)+\phi\right\}\right] \equiv \sum_{n=-\infty}^{+\infty} J_{n}\left(\frac{k_{x} v_{R}}{\omega_{c}}\right) \exp \left(-i n\left\{\omega_{c}\left(t^{\prime}-t\right)+\phi\right\}\right)
$$

Inserting this in (6) and integrating yields,

$$
x_{1}^{\prime}=A \exp \left[i\left(\omega-k_{z} v_{z}\right)\left(t^{\prime}-t\right)\right] \sum_{n=-\infty}^{+\infty} \frac{J_{n}\left(\frac{k_{x} v_{R}}{\omega_{c}}\right) \exp \left(-i n\left\{\omega_{c}\left(t^{\prime}-t\right)+\phi\right\}\right)}{\left[\omega_{c}^{2}-\left(\omega-k_{z} v_{z}-n \omega_{c}\right)^{2}\right]} .
$$

The rf perturbation becomes very large when $\left(\omega-k_{z} v_{z}\right)$ approaches $(n \pm 1) \omega_{c}$, i.e., strong interactions occur between the rf electric field and the electron when the Doppler-shifted frequency approaches a cyclotron harmonic frequency. These cyclotron harmonic interactions result from two factors: the d-c rotational energy of the electron, and the fact that the rf electric field has a transverse variation $\left(k_{x} \neq 0\right)$. This gives rise to space harmonics of the electric field over the direction of the electron motion, and hence strong interactions. As $k_{x}$ or $v_{R}$ decrease, the higherorder interactions will become progressively weaker. When $\left(k_{x} v_{R} / \omega_{c}\right)$ is zero only the fundamental cyclotron resonance predicted by cold plasma theory remains.

\subsection{Multiparticle Treatment}

The next step is to develop a multiparticle treatment in which the space-charge forces due to the presence of many electrons couple their motions and lead to a dispersion relation. The first attempt to do this appears to have been due to Malmfors [1950], who studied the stability of crossedfield microwave devices. His work contained an algebraic error that was corrected by Gross [1951], whose work was in turn taken up and extended by Sen [1952], using the Laplace transform approach 
initiated by Landau [1946]. The subject was discussed again in a series of papers, the most complete of these being that due to Bernstein [1958] which corrected several errors in previous analyses.

In the averaging over the individual particle motions, two approaches are possible. A tensor expression for the rf current can be derived and substituted in Maxwell's equation [Bernstein, 1958; Stix, 1962] or an approximation can be made at the outset which allows us to work with scalar quantities and simplifies considerably the tedious integrations involved. This is the quasistatic, or "slow-wave," approximation, and consists in replacing the rf electric field $\vec{E}$, by the gradient of a potential, $(-\nabla \Phi)$. The approximation amounts to considering only waves for which

$$
\left|k_{x}\right|^{2}+\left|k_{z}\right|^{2} \gg \frac{\omega^{2}}{c^{2}}\left|\epsilon_{i j}\right|
$$

where $\epsilon_{i j}$ are elements of the tensor permittivity of the plasma [Stix, 1962]. We assume, without loss of generality, variations in the $x$ and $z$ directions only, and $c$ is the speed of light. It is also implied that $\vec{E}$ and $\vec{k}$ are parallel. Poisson's equation, for the rf space charge density is then,

$$
-i\left(k_{x} E_{x}+k_{z} E_{z}\right)=\left(k_{x}^{2}+k_{z}^{2}\right) \Phi=\frac{\rho_{1}}{\epsilon_{0}} .
$$

To find a dispersion relation, it is now necessary to determine $\rho_{1}$ in terms of the rf electric fields in the system. The basic steps in the analysis will now be indicated without specifying the unperturbed electron velocity distribution.

\subsection{Determination of the RF Charge Density}

Liouville's theorem concerning particle number density, $f\left(x, y, z, v_{x}, v_{y}, v_{z}\right)$ in phase space states that the number density of particles in a volume element is conserved as this volume moves in phase space. Hence, if $f_{0}$ and $f_{1}$ are the d-c and $\operatorname{rf}$ components of $f$, we have

$$
\frac{d f_{0}}{d t}+\frac{d f_{1}}{d t}=0
$$

Written out in full, this is

$$
\left[\frac{\partial f_{0}}{\partial t}+\vec{v} \cdot \nabla f_{0}+\eta \vec{v} \times \vec{B}_{0} \cdot \frac{\partial f_{0}}{\partial \vec{v}}\right]+\left[\frac{\partial f_{1}}{\partial t}+\vec{v} \cdot \nabla f_{1}+\eta\left(\vec{E}+\vec{v} \times \vec{B}_{0}+\vec{v} \times \vec{B}_{1}\right) \cdot \frac{\partial f}{\partial \vec{v}}\right]=0
$$

To obtain $f_{1}$ correct to first order, we must solve

$$
\left(\frac{d f_{1}}{d t}\right)_{0}=-\eta \vec{E} \cdot \frac{\partial f_{0}}{\partial \vec{v}}
$$

where the time-differentiation is along the unperturbed electron trajectory, and the rf magnetic field term can be neglected in a quasi-static analysis. Integration of (13) gives

$$
f_{1}(t)=-\eta \int_{t_{0}}^{t} \vec{E}^{\prime} \cdot \frac{\partial f_{0}}{\partial \vec{v}^{\prime}} d t^{\prime}+f_{1}\left(t_{0}\right)
$$

Next, assume that $f_{1}$ is of the form $F \exp i\left(\omega t-k_{x} x-k_{z} z\right)$, and that $\overrightarrow{E^{\prime}}$ is given by $\vec{E} \exp i\left(\omega t^{\prime}\right.$ $-k_{x} x^{\prime}-k_{z} z^{\prime}$ ). Substitution from (3) and (4) in (14) yields in cylindrical coordinates, 


$$
\begin{array}{r}
F\left(v_{z}, v_{R}, \phi\right)=-\eta \int_{-\infty}^{t} \exp i\left[\left(\omega-k_{z} v_{z}\right)\left(t^{\prime}-t\right)-\left(\frac{2 k_{x} v_{R}}{\omega_{c}}\right) \sin \left\{\frac{\omega_{c}}{2}\left(t^{\prime}-t\right)\right\} \cos \left\{\frac{\omega_{c}}{2}\left(t^{\prime}-t\right)+\phi\right\}\right] \\
\times\left[E_{z} \frac{\partial f_{0}}{\partial v_{z}}+E_{x} \frac{\partial f_{0}}{\partial v_{R}} \cos \left\{\omega_{c}\left(t^{\prime}-t\right)+\phi\right\}\right] d t^{\prime} .
\end{array}
$$

A lower limit of $t_{0}=-\infty$ has been taken, assuming that there is no initial bunching of the electrons, i.e., $f_{1}\left(t_{0}\right) \rightarrow 0$ as $t_{0} \rightarrow-\infty$. This is equivalent to assuming that a small loss component is present. The limit is also justifiable as the only one yielding periodic solutions in $\Phi$ [Stix, 1962].

To obtain $\rho_{1}, F$ must be integrated over $v_{z}, v_{R}$, and $\phi$. The integration over $\phi$ is between limits of 0 and $2 \pi$ and may be accomplished by means of the substitutions of $\tau$ for $\left(t^{\prime}-t\right)$ and $\psi$ for $\left[\phi+\left(\omega_{c} \tau / 2\right)\right]$, and use of the Bessel function identity [Bateman Manuscript Project, 1953]

$$
J_{n}\left(\frac{2 k_{x} v_{R}}{\omega_{c}} \sin \frac{\omega_{c} \tau}{2}\right) \equiv \frac{i^{n}}{2 \pi} \int_{0}^{2 \pi} \exp \left[-i\left(\frac{2 k_{x} v_{R}}{\omega_{c}} \sin \frac{\omega_{c} \tau}{2}\right) \cos \psi\right] \cos n \psi d \psi
$$

The resulting function, $G$, is given by

$$
\begin{aligned}
G\left(v_{z}, v_{R}\right)=-2 \pi \eta \int_{-\infty}^{0} \exp \left[i\left(\omega-k_{z} v_{z}\right) \tau\right]\left[E_{z} \frac{\partial f_{0}}{\partial v_{z}} J_{0}\left(\frac{2 k_{x} v_{R}}{\omega_{c}} \sin \frac{\omega_{c} \tau}{2}\right)\right. & \\
& \left.-i E_{x} \frac{\partial f_{0}}{\partial v_{R}} \cos \left(\frac{\omega_{c} \tau}{2}\right) J_{1}\left(\frac{2 k_{x} v_{R}}{\omega_{c}} \sin \frac{\omega_{c} \tau}{2}\right)\right] d \tau .
\end{aligned}
$$

To complete the integration, a further Bessel function identity and its differential form are required [Bateman Manuscript Project, 1953]

$$
\begin{gathered}
J_{0}\left(\frac{2 k_{x} v_{R}}{\omega_{c}} \sin \frac{\omega_{c} \tau}{2}\right) \equiv \sum_{n=0}^{\infty} \Delta_{n} J_{n}^{2}\left(\frac{k_{x} v_{R}}{\omega_{c}}\right) \cos n \omega_{c} \tau, \\
\cos \left(\frac{\omega_{c} \tau}{2}\right) J_{1}\left(\frac{2 k_{x} v_{R}}{\omega_{c}} \sin \frac{\omega_{c} \tau}{2}\right) \equiv\left(\frac{2 \omega_{c}}{k_{x} v_{R}}\right) \sum_{n=1}^{\infty} n J_{n}^{2}\left(\frac{k_{x} v_{R}}{\omega_{c}}\right) \sin n \omega_{c} \tau,
\end{gathered}
$$

where $\Delta_{0}=1 ; \Delta_{n}=2, n \neq 0$. Using these and again assuming that a slight loss component is present, we obtain

$G\left(v_{z}, v_{R}\right)=-2 \pi \eta \Phi\left[k_{z} \frac{\partial f_{0}}{\partial v_{z}} \sum_{n=0}^{\infty} \frac{\Delta_{n}\left(\omega-k_{z} v_{z}\right) J_{n}^{2}\left(\frac{k_{x} v_{R}}{\omega_{c}}\right)}{\left(\omega-k_{z} v_{z}\right)^{2}-\left(n \omega_{c}\right)^{2}}+\left(\frac{2 \omega_{c}^{2}}{v_{R}}\right) \frac{\partial f_{0}}{\partial v_{R}} \sum_{n=1}^{\infty} \frac{n^{2} J_{n}^{2} \frac{k_{x} v_{R}}{\omega_{c}}}{\left(\omega-k_{z} v_{z}\right)^{2}-\left(n \omega_{c}\right)^{2}}\right]$

An equivalent expression to this has been derived by Harris [1959, 1961]. Its value lies in the fact that when the unperturbed velocity distribution, $f_{0}$, is specified, the $\mathrm{rf}$ charge density is immediately obtainable from

$$
\rho_{1}=e \int_{-\infty}^{+\infty} d v_{z} \int_{0}^{\infty} v_{R} G\left(v_{z}, v_{R}\right) d v_{R}
$$

If several species of particles are present their space-charge contributions are additive. 


\subsection{Dispersion Relations for Oblique Propagation $\left(k_{x} \neq 0, k_{z} \neq 0\right)$}

It is instructive to consider first a uniform electron beam having a velocity distribution of the form

$$
f_{0}=\frac{n_{0}}{2 \pi v_{R 0}} \delta\left(v_{R}-v_{R 0}\right) \delta\left(v_{z}-v_{z 0}\right)
$$

The $\delta$-functions imply that the beam has a translational velocity, $v_{z 0}$, and that all electrons have the same speed of gyration, $v_{R 0}$, about the field lines. Substitution of (21) in (19) and (20), and combination of the result with (10) leads to the dispersion relation for the cyclotron harmonic waves on the beam,

$$
\begin{array}{r}
0=k_{x}^{2}\left[1-\omega_{p}^{2} \sum_{n=1}^{\infty}\left\{\frac{n^{2} \frac{\partial}{\partial \mu}\left(J_{n}^{2}(\mu)\right)}{\left(\frac{\mu}{2}\right)\left[\left(\omega-k_{z} v_{z 0}\right)^{2}-\left(n \omega_{c}\right)^{2}\right]}\right\}\right] \\
+k_{z}^{2}\left[1-\omega_{p}^{2} \sum_{n=0}^{\infty} \Delta_{n} J_{n}^{2}(\mu)\left\{\frac{\left(\omega-k_{z} v_{z 0}\right)^{2}+\left(n \omega_{c}\right)^{2}}{\left[\left(\omega-k_{z} v_{z 0}\right)^{2}-\left(n \omega_{c}\right)^{2}\right]^{2}}\right\}\right],
\end{array}
$$

where $\omega_{p}$ is the electron plasma frequency corresponding to the beam density, and $\mu$ has been written for $\left(k_{x} v_{v_{0}} / \omega_{c}\right)$. It will be noted that this is the parameter previously encountered in the single-particle model of (8). As $\mu \rightarrow 0$ we retrieve the Doppler-shifted cold-plasma dispersion relation

$$
0=k_{x}^{2}\left[1-\frac{\omega_{p}^{2}}{\left(\omega-k_{z} v_{z 0}\right)^{2}-\omega_{c}^{2}}\right]+k_{z}^{2}\left[1-\frac{\omega_{p}^{2}}{\left(\omega-k_{z} v_{z 0}\right)^{2}}\right] .
$$

Next, consider an isotropic Maxwellian electron velocity distribution such that

$$
f_{0}=n_{0}\left(\frac{m}{2 \pi \kappa T_{e}}\right)^{3 / 2} \exp \left[\frac{-m\left(v_{z}^{2}+v_{R}^{2}\right)}{2 \kappa T_{e}}\right]
$$

Substitution of (24) in (19) and (20) and combination with (10) will again lead to a dispersion relation. Alternatively, (22) may be regarded as the basic dispersion expression which is then written with (24) as

$$
\begin{aligned}
& k_{x}^{2}+k_{z}^{2}=\frac{\omega_{p}^{2}}{\lambda}\left(\frac{m}{2 \pi \kappa T_{e}}\right)^{1 / 2}\left[k _ { x } ^ { 2 } \int _ { 0 } ^ { \infty } \sum _ { n = 1 } ^ { \infty } 2 n ^ { 2 } \frac { \partial } { \partial \mu } \left(J_{n}^{2}(\mu) \exp \left(\frac{\mu^{2}}{2 \lambda}\right) d \mu \int_{-\infty}^{+\infty} \frac{\exp \left(-\frac{m v_{z}^{2}}{2 \kappa T_{e}}\right) d v_{z}}{\left(\omega-k_{z} v_{z}\right)^{2}-\left(n \omega_{c}\right)^{2}}\right.\right. \\
& \left.\quad+k_{z}^{2} \int_{0}^{\infty} \sum_{n=0}^{\infty} \Delta_{n} J_{n}^{2}(\mu) \exp \left(-\frac{\mu^{2}}{2 \lambda}\right) \mu d \mu \int_{-\infty}^{+\infty} \exp \left(-\frac{m v_{z}^{2}}{2 \kappa T_{e}}\right)\left\{\frac{\left(\omega-k_{z} v_{z}\right)^{2}+\left(n \omega_{c}\right)^{2}}{\left[\left(\omega-k_{z} v_{z}\right)^{2}-\left(n \omega_{c}\right)^{2}\right]^{2}}\right\} d v_{z}\right],
\end{aligned}
$$

where $\lambda$ has been written for $\left(k_{x}^{2} \kappa T_{e} / m \omega_{c}^{2}\right)$ or $\left(k_{x} R\right)^{2}$; and hence, $R$ is the radius of gyration of the average thermal electron.

The integration with respect to $\mu$ is accomplished using the identity [Bateman Manuscript Project, 1953]

$$
\int_{0}^{\infty} J_{n}^{2}(\mu) \exp \left(-\frac{\mu^{2}}{2 \lambda}\right) \mu d \mu=\lambda \exp (-\lambda) I_{n}(\lambda)
$$


After some manipulation we arrive at the expression

$$
\begin{aligned}
k_{x}^{2}+k_{z}^{2}=\omega_{p}^{2}\left(\frac{m}{2 \pi \kappa T_{e}}\right)^{1 / 2} & k_{x}^{2} \sum_{n=1}^{\infty}\left(\frac{2 n^{2}}{\lambda}\right) \exp (-\lambda) I_{n}(\lambda) \int_{-\infty}^{+\infty} \frac{\exp \left(\frac{-m v_{z}^{2}}{2 \kappa T_{e}}\right) d v_{z}}{\left(\omega-k_{z} v_{z}\right)^{2}-\left(n \omega_{c}\right)^{2}} \\
& \left.+\left(\frac{m}{\kappa T_{e}}\right) \sum_{n=0}^{\infty} \triangle_{n} \exp (-\lambda) I_{n}(\lambda) \int_{-\infty}^{+\infty} \frac{k_{z} v_{z}\left(\omega-k_{z} v_{z}\right) \exp \left(-\frac{m v_{z}^{2}}{2 \kappa T_{e}}\right)}{\left(\omega-k_{z} v_{z}\right)^{2}-\left(n \omega_{c}\right)^{2}} d v_{z}\right] .
\end{aligned}
$$

The integrand of (25) regarded as a function of $v_{R}$ (or $\mu$ ) has no poles. Consequently, no Landau damping terms appear in (27) due to the electron motions transverse to the magnetic field. The physical reason for their absence is that all electrons retrace their paths periodically, at the cyclotron frequency. Hence, there is no phase-mixing. However, (27) indicates that there can be Landau damping due to the velocity distribution in the $z$-direction, except for the special case of purely perpendicular propagation $\left(k_{z}=0\right)$. Oblique propagation has been dealt with by Stix [1962] and we shall not discuss it further other than to remark that the damping will only by negligible if the condition $\left(\omega-n \omega_{c}\right) \gg k_{z}\left(2 \kappa T_{e} / m\right)^{1 / 2}$ is satisfied. This implies that for modes corresponding to $n>1$ propagation must be almost perpendicular to the magnetic field. By far the most interesting case, then, is that of perpendicular propagation which we shall now discuss in more detail.

\subsection{Dispersion Relations for Perpendicular Propagation $\left(k_{x} \neq 0, k_{z}=0\right)$}

For the two velocity distributions studied, (22) and (27) give

$$
\mathrm{l}=\frac{\omega_{p}^{2}}{\omega_{c}^{2}} \sum_{n=1}^{\infty} \frac{\frac{\partial}{\partial \mu}\left(J_{n}^{2}(\mu)\right)}{\left(\frac{\mu}{2}\right)\left[\left(\frac{\omega}{n \omega_{c}}\right)^{2}-1\right]}, \quad \mathrm{I}=\frac{\omega_{p}^{2}}{\omega_{c}^{2}} \sum_{n=1}^{\infty} \frac{\exp (-\lambda) I_{n}(\lambda)}{\left(\frac{\lambda}{2}\right)\left[\left(\frac{\omega}{n \omega_{c}}\right)^{2}-1\right]} .
$$

It may be remarked here that the series representation is not the most satisfactory for computation. An extremely useful integral expression is derived in an appendix to the paper.

The behavior of the two expressions of (28) differ considerably. The first is shown plotted in figures $2 \mathrm{a}, \mathrm{b}, \mathrm{c}$, and $\mathrm{d}$ for different values of $\left(\omega_{p}^{2} / \omega_{c}^{2}\right)$. Each passband demonstrates a resonance $\left(k_{x} \rightarrow \infty\right)$ at the appropriate cyclotron harmonic frequency. Cutoffs $\left(k_{x} \rightarrow 0\right)$ occur at $(n+1) \omega_{c}$, and at the point $X$ corresponding to the upper hybrid frequency $\left(\omega_{p}^{2}+\omega_{c}^{2}\right)^{1 / 2}$ familiar from coldplasma theory. The oscillatory nature of the dispersion relation in each passband stems from the nature of $\partial / \partial \mu\left[J_{n}^{2}(\mu)\right]$ which shows all of the zeros of $J_{n}(\mu)$ and $J_{n}^{\prime}(\mu)$ as indicated in the figures. At low values of $\left(\omega_{p}^{2} / \omega_{c}^{2}\right)$ individual modes do not couple. For larger values, such coupling can occur and gaps in the propagation appear. In these gaps the solution for $\omega$ obtained for real values of $\mu$ is complex, and indicates the existence of growing waves. This implies that the system is unstable [Crawford and Tataronis, 1965]. We shall return later to this extremely important point.

Figure 3 shows dispersion curves for the Maxwellian distribution. The same resonances and cutoffs are indicated as in figures $2 \mathrm{a}, \mathrm{b}, \mathrm{c}$, and $\mathrm{d}$ but no coupling of the individual modes occurs and the system is absolutely stable.

So far, we have not discussed the effect of collisions on the otherwise undamped perpendicular propagation of cyclotron harmonic waves. It has been shown elsewhere [Crawford, Kino, and Weiss, 1964b] that electron/neutral collisions occurring with frequency $\nu$ can be taken into account in (28) by replacing $\omega$ by $(\omega-i \nu)$ and $\omega_{p}^{2}$ by $\omega_{p}^{2}\left(1-i \frac{\nu}{\omega}\right)$. To obtain a simple order of magnitude estimate for the attenuation, it may be assumed that $k_{x}$ is some function, $K$, of $(\omega-i \nu)$. Evaluating 

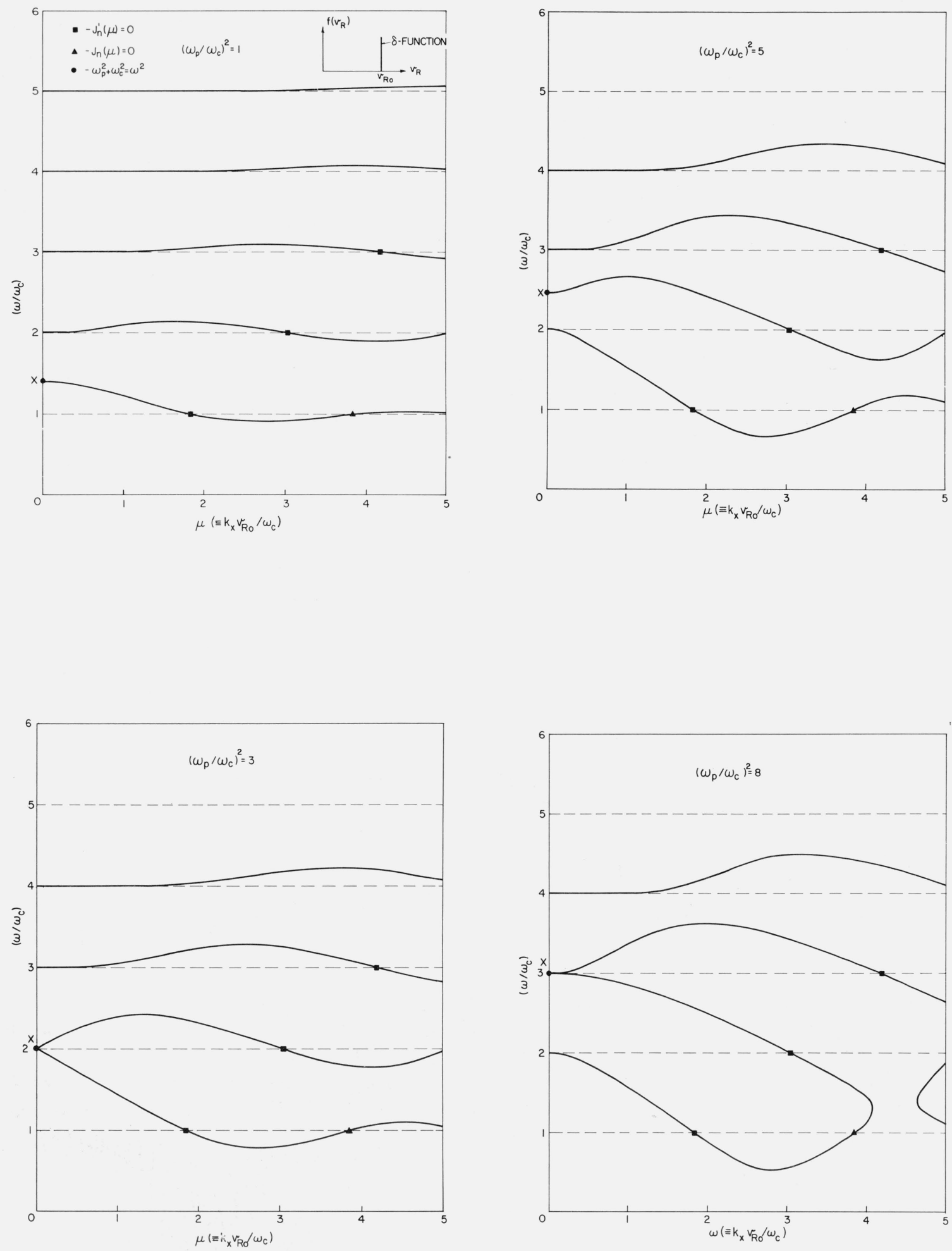

FIGURE 2. Dispersion characteristics for perpendicular propagation of cyclotron harmonic waves-monoenergetic transverse velocity distribution. 
FIGURE 3. Dispersion characteristics for perpendicular propagation of cyclotron harmonic waves-Maxwellian transverse velocity distribution.

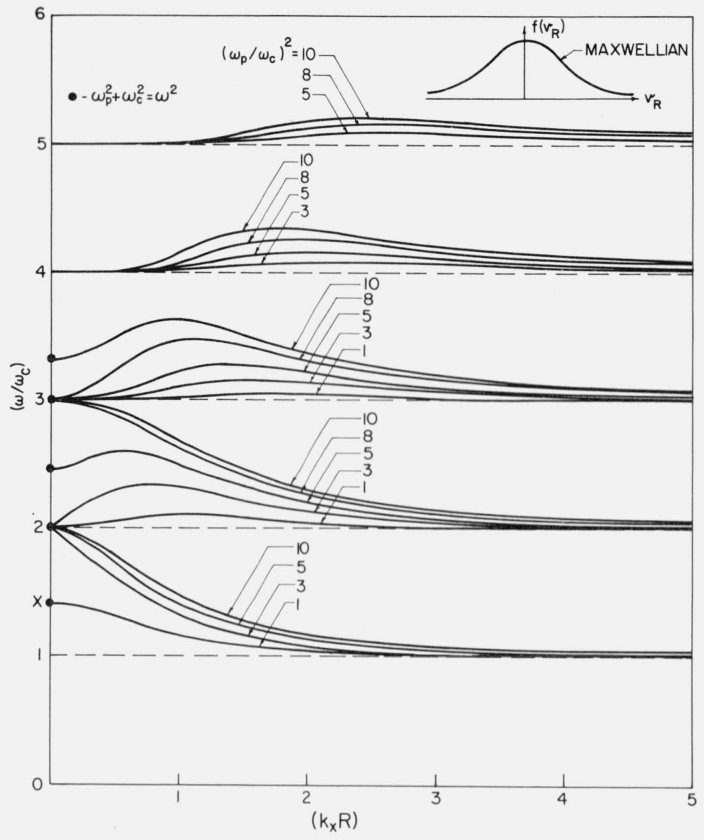

the real and imaginary parts of $k_{x}$, yields

$$
k_{x r}+i k_{x i} \approx K(\omega)-i \nu \frac{\partial k_{x r}}{\partial \omega} .
$$

The group velocity, $v_{g}$, may be introduced to write $k_{x i}$ in the form $\left(-\nu / v_{g}\right)$ which represents attenuation/unit length. It is a feature of the computed curves of figures $2 \mathrm{a}, \mathrm{b}, \mathrm{c}, \mathrm{d}$, and 3 that $v_{g}$ generally lies below the electron thermal velocity and decreases rapidly with increasing harmonic number. This means that the collision damping of perpendicular propagation may be strong if $\nu$ is not small, and will always limit the number of harmonics observable.

\section{Experiments}

\subsection{General Comments}

In this section we shall describe some of the experiments which confirm the existence of cyclotron harmonic waves. These are dealt with in detail elsewhere [Crawford, 1964b], and we shall only indicate briefly the nature of the phenomena involved. It should be emphasized that although the observations date back to 1959 [Wharton, 1960], their explanation was not then clear, and it was in 1963 [Canobbio and Croci, 1964; Tanaka, Mitani, and Kubo, 1963] that the suggestion was first advanced that these waves might be responsible for certain cyclotron harmonic noise phenomena in plasmas. Since these were the subject of the earliest studies we shall begin our brief review with some comments on them.

\subsection{Studies of Plasma Emission}

Although the first observations of radiation at $\left(n \omega_{c}\right)$ seem to have been those of Wharton [1960], in which noise emission from a pulsed mirror machine was studied, the first extensive series of experiments was that of Landauer [1961, 1962, 1964], using a PIG discharge as shown 

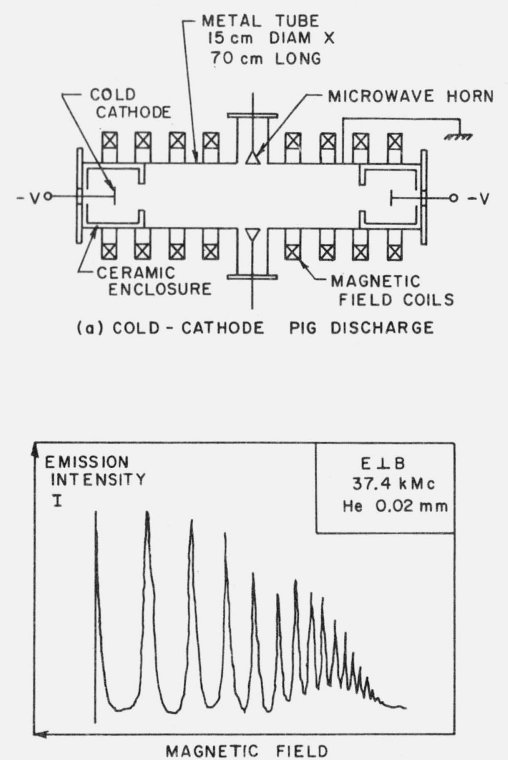

(b) TYPICAL NOISE EMISSION RECORD

FIGURE 4. Cyclotron harmonic radiation from a PIG discharge [after Landauer, 1961, 1962, 1964].

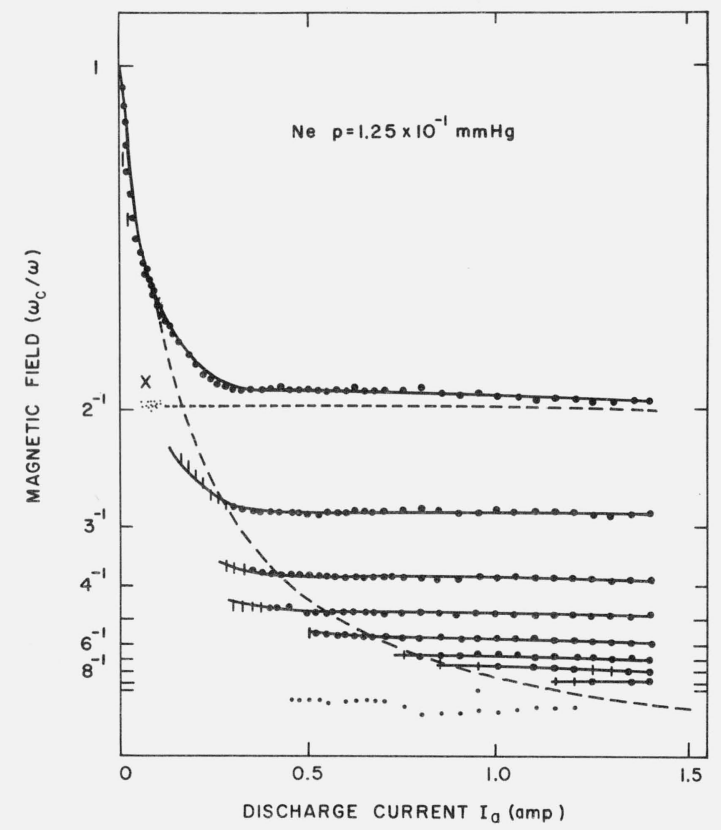

FiguRE 5. Dispersion characteristics of cyclotron harmonic radiation from a plasma [after Tanaka et al., 1963].

in figure 4. These indicated anomalously strong noise components up to the 45th harmonic, and fitting the relation $\left(\omega / \omega_{c}\right)=n$ to better than 2 percent. The most intense radiation was measured for the polarization $\vec{E} \perp \vec{B}$, but harmonic emission was also obtained for $\vec{E} \| \vec{B}$. We may remark here that this latter result may easily be predicted from examination of the dielectric tensor obtained without making the quasi-static assumption. This indicates that the ordinary wave, $\vec{E} \| \vec{B}, \vec{k} \perp \vec{B}$ should also show cyclotron harmonic resonances [Stix, 1962; Kubo et al., 1964].

The major conclusions of Landauer have been confirmed by Dreicer [1964], in a PIG discharge, by Lustig et al. [1964]; Mitani and Kubo [1961]; Tanaka et al. [1963a, b], and Mitani et al. [1964a, b]. These latter worked with hot-cathode discharges and were the first to remark that there are significant discrepancies from the relation $\left(\omega / \omega_{c}\right)=n$ for maximum noise emission. Typical results are given in figure 5 .

The reasons for the very high noise production in PIG discharges are clear from the discussion of section 2.5. Such a discharge contains streams of electrons having energy equal to the operating voltage, and presents ideal conditions for transverse instabilities or strong beam/ plasma interactions with the cyclotron harmonic modes.

It might be argued that strong directional velocity components were not present in some hotcathode discharge experiments where many harmonics were also observed [Lustig et al., 1964; Mitani and Kubo, 1961; Tanaka and Kubo, 1964; Kubo et al., 1964]. It was always noted in these studies, however, that noise emission was highest near the cathode end of the tube and at points where the plasma entered the magnetic field. The results imply, then, that streams of primary electrons entering from the cathode region, and acquiring high transverse velocity components, are effective in producing cyclotron harmonic radiation.

As further support for the hypothesis concerning the effectiveness of charged particle beams in exciting cyclotron harmonic resonances, we may refer to radiation measurements carried out in the millimeter pressure range where such streams would be rapidly dispersed. Experimental results along these lines were reported by Bekefi et al. [1961a, b; Fields et al., 1961; Hirshfield and Brown, 1961; Brown and Bekefi, 1962] and have been substantially confirmed by Tanaka and 
Kubo [1964]. They show characteristic noise radiation dependent on the gas: in helium and hydrogen, the effective radiation temperature corresponds to the electron temperature, but in neon, argon, and xenon narrow peaks in the radiation temperature occur, centered on $\omega_{c}$. These results can be ascribed to strongly non-Maxwellian electron velocity distributions in the lastmentioned gases, where the electron-neutral collision cross section decreases with energy in the energy range appropriate to the measurements [Ohara, 1962, 1963]. The conclusion to be drawn, then, is that beam-type or "humped" velocity distributions are capable of exciting strong cyclotron harmonic waves.

The best test of this, of course, is to use an electron beam to produce the plasma. This ensures the presence of both beam and plasma. Two experiments along these lines have been reported [Bekefi and Hooper, 1964; Gruber et al., 1964]. In the first of these, the beam and the discharge tube were coaxial with the magnetic field, and the gun was only partially immersed, so that about 15 percent of the longitudinal beam energy was converted into transverse rotation. Emission at $\omega_{c}$ due to the beam electrons alone could be observed, appropriately Doppler-shifted in frequency. When mercury-vapor was present, however, the radiated signal increased by $35 \mathrm{~dB}$ and similar Doppler-shifted components could be seen up to the fourth harmonic. Radiation from a beamproduced plasma in helium up to the 15th harmonic was studied by Gruber et al. [1964], who examined the radial and longitudinal amplitude variations of the output signals by means of a movable probe. They presented results showing fast-wave growth of the 8th to 10th cyclotron harmonics at about $11 \mathrm{~dB}$ per wavelength in the direction of the beam, and with rapid radial fall-off.

\subsection{Absorption Studies}

Having discussed experiments in which noise emission only has been studied, we now turn to attempts to excite resonance behavior with an external signal. Bekefi et al. [1962] reported work on a hot-cathode helium discharge in a cylindrical cavity excited in the $\mathrm{TE}_{011}$ mode, and immersed in an axial magnetic field. It was found that for $\omega_{p}^{2}<<\omega^{2}$, resonant absorption occurred at $\omega_{c}$, and that for $\omega_{p}^{2} \approx \omega^{2}$, weak absorption of up to the first eight cyclotron harmonics occurred.

Comparisons of absorption and emission under identical experimental conditions have been made by Tanaka and Kubo [1964]. Weak absorption occurred up to the 5th harmonic, whereas noise radiation was appreciable up to the 8 th harmonic. This is to be expected since we are dealing with radiation orders of magnitude higher than that appropriate to thermal equilibrium. Reciprocity between emission and absorption would occur only under these special conditions.

Further absorption studies, both theoretical and experimental, have been made by Buchsbaum and Hasegawa [1964] to explain an effect first remarked by Kubo et al. [1964]: the noise radiation can show series of subsidiary resonances lying between the main harmonic resonance. With increasing discharge current, these successively approach the harmonic resonances, increasing in magnitude as they do so, and disappear on reaching them. The subsidiary resonances were explained as being due to the presence of radial electron density variation in the plasma. They are analogous to the Tonks-Dattner resonances mentioned in section 1, but are "trapped" in the center of the column, rather than in the region close to the wall. An alternative source of subsidiary resonances due to wave interference has been studied recently by Harp [1965].

By far the most interesting observations from the point of view of ionospheric propagation were the results obtained with the "Alouette" satellite [Calvert and Goe, 1963; Lockwood, 1963]. In these, the vehicle carried a transmitter/antenna system giving a pulsed variable frequency signal. Strong resonances were found to occur within 1 percent of the calculated local cyclotron harmonic frequencies, up to about the 10th cyclotron harmonic. Prolonged ringing was observed at these frequencies, persisting for many periods after the end of the transmitter pulse. Since no such effect has been seen from the ground, we may deduce that the effect was local to the transmitter.

It was suggested that since the perpendicular propagation of cyclotron harmonic modes is not Landau damped, and collision frequencies in the ionosphere are relatively low, a ringing 
effect is to be expected near $n \omega_{c}$, where the group velocity is low and the energy is not transported rapidly away from the transmitter [Crawford, Kino, and Weiss, 1964a]. This explanation has been developed by Fejer and Calvert [1964], and Sturrock [1965], who have estimated the damping rate of the resonances.

\subsection{Propagation Studies}

Although the results described so far provide strong support for the cyclotron harmonic wave picture, in that they are all consistent with waves propagating nearly normal to the magnetic field, one very important observation is lacking. This is a direct measurement of transmission across the magnetic field between two antennas. Such an experiment was carried out for the first time in 1964 [Crawford, Kino, and Weiss, 1964a, 1964b]. In the original experiment two Langmuir probes, parallel to each other and to a static magnetic field, and situated a few millimeters apart in a hot-cathode mercury-vapor discharge were used as emitting and receiving antennas respectively for a $400 \mathrm{MHz}$ signal. Peaks in the received signal were observed for certain combinations of discharge current and magnetic field. Typical data are shown in figure 6a.

A direct comparison with the theoretical curves of figure 3 cannot be made since $\omega_{c}$ and $\omega_{p}$ (proportional to $I_{a}^{1 / 2}$ ) were the experimental variables. It is instructive, however, to compute from the theoretical dispersion relation the variation of $\left(\omega_{c}^{2} / \omega^{2}\right)$ with $\left(\omega_{p}^{2} / \omega^{2}\right)$. This is indicated in figures $7 \mathrm{a}, \mathrm{b}, \mathrm{c}$. The coupling of the cyclotron harmonic frequencies and the cold plasma upper hybrid resonance are clearly indicated as the parameter $\left(v_{p} / v_{T}\right)$ is varied. There is striking agreement between the general shapes of these curves and the experimental curves of figures 5 and $6 a$.

If the radial wavelength were constant in the experiment the experimental points could be compared directly to the theory. However, the nonuniform density in the cylindrical plasma column employed causes the modes to be trapped internally so that the radial wavelength varies with current. This is clearly indicated by the presence of two distinct sets of resonances in figure $6 \mathrm{a}$. These are for two different trapped modes. When a more uniform discharge is produced by $\mathrm{rf}$ the transmission resonances are much cleaner. A typical record is shown in figure $6 \mathrm{~b}$ and indicates the presence of only one set of peaks. We can, however, indicate on the plot of figure 6a the approximate location of the upper hybrid resonance frequency obtained by an independent method [Crawford et al., 1963]. This is shown dotted in figure 6a, and reinforces our belief in the validity of the cyclotron harmonic wave explanation of the transmission resonances.
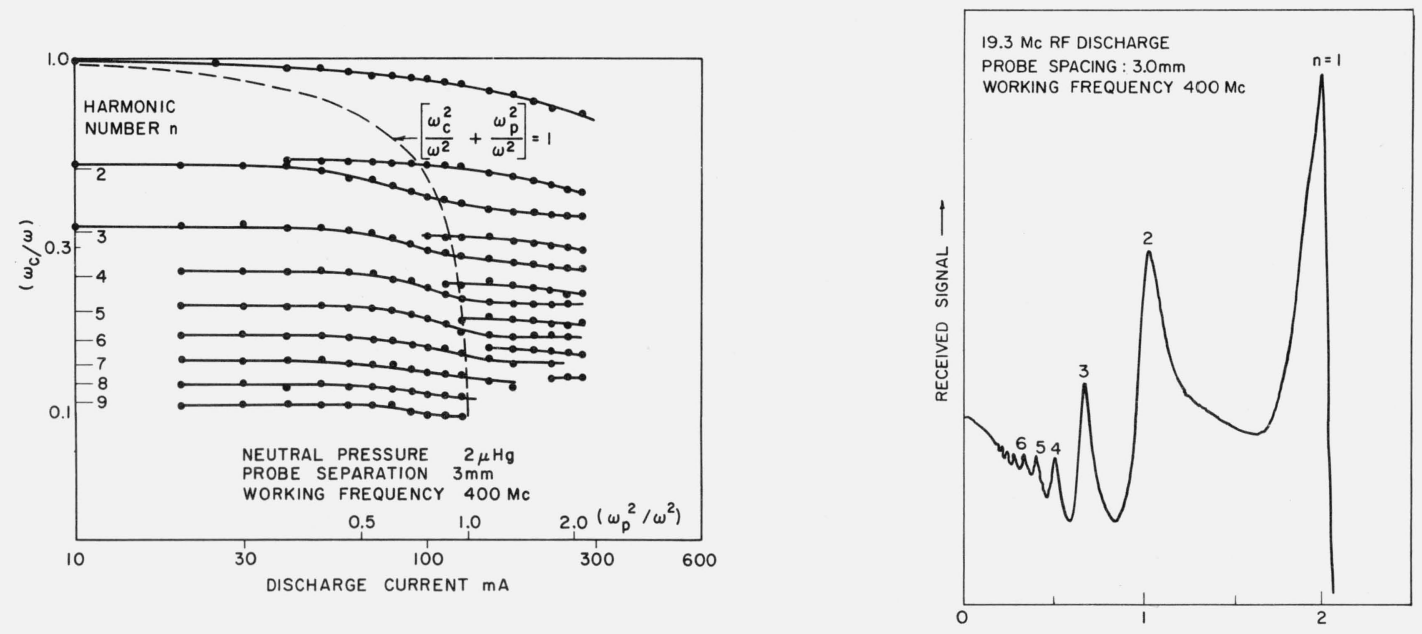

FIGURE 6. Experimental observations of perpendicular propagation.

(a) Dispersion characteristics [after Crawford, Kino, and Weiss 1964].
Wranersion

(b) Transmission record for an rf discharge (the abscissa may be calibrated in terms of $\left.\left(\omega_{c} / \omega\right)\right)$. 

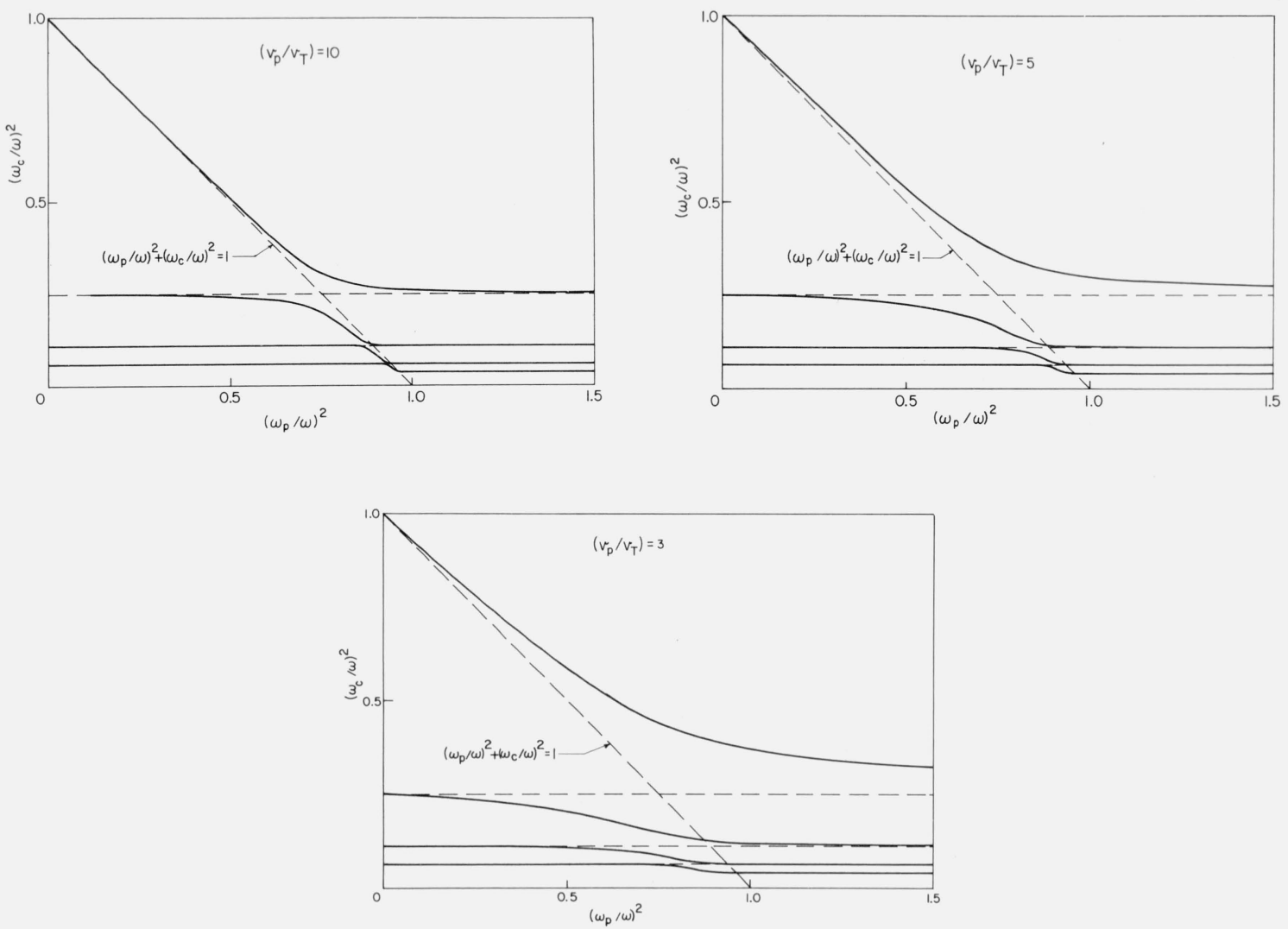

FIGURE 7. Alternative representation of the perpendicular propagation dispersion characteristics of figure 3 for a Maxwellian transverse velocity distribution $\left[\left(\mathrm{v}_{\mathrm{p}}=\left(\omega / \mathrm{k}_{\mathrm{x}}\right)\right.\right.$, $\left.\mathrm{v}_{\mathrm{T}}=\left(\kappa \mathrm{T}_{\mathrm{e}} / \mathrm{m}\right)^{\mathbf{1} / 2}\right]$.

\section{Discussion}

The complicated single-particle analysis required to demonstrate the existence of cyclotron harmonic waves has probably been partly responsible for the fact that their existence has been established experimentally almost by accident, rather than as the result of deliberate searches. Of the studies described, only those concerned with transmission were made specifically to compare with the form of the theoretical dispersion relation.

Now that their existence has been established, the importance of these waves can be expected to grow. First, there is the possibility of applying them in practical laboratory microwave amplifiers and oscillators [Crawford and Kino, 1964]. As they are slow waves, interaction with an electron beam directed either along the magnetic field, or across it, will cause spatially growing waves. Alternatively, a beam with internal motions can interact directly with slow or fast wave circuits to produce gain. Such a beam could be produced in nature by a charged particle stream entering a region of increasing magnetic field. Second, taking the opposite point of view, the explanation of many plasma noise phenomena, including extraterrestrial cyclotron emission, may be better understood in terms of cyclotron harmonic wave instabilities [Twiss, 1958; Schneider, 1959; Bekefi, Hirshfield, and Brown 196la and 1961b]. A third region of application is in diagnostics. The "Alouette" ringing experiments suggests that the resonances could be used for measuring magnetic field accurately. Attenuation measurements could be used to determine collision frequencies, and in principle it should be possible to determine electron density and temperature from transmission measurements between two antennas. 
All that has been said in this paper about electron effects can be extended to the positive ions. Consequently, it is not surprising that experimental observations have already been made of ion cyclotron harmonic emission from thermonuclear fusion study plasmas [Bazhanova et al., 1962; Pistunovich and Shafranov, 1961; Crawford, 1964c]. The possibility that ion cyclotron waves might be useful for plasma heating immediately suggests itself.

The author wishes to acknowledge the assistance of J. Tataronis with the computations, and many fruitful discussions of the theory with Dr. G. S. Kino.

\section{Appendix. Integral Representation of the Dispersion Relation for Perpendicular Propagation $\left(k_{z}=0\right)$}

A convenient integral form for computation may be derived from (17), (20), and (21) by carrying out the velocity integrations first. This leads to

$$
1=\frac{\omega_{p}^{2}}{\omega_{c}^{2}} \int_{-\infty}^{0} \exp i \Omega \theta \sin \theta J_{0}\left(2 \mu \sin \frac{\theta}{2}\right) d \theta
$$

where $\theta$ has been written for $\left(\omega_{c} \tau\right)$, and $\Omega$ for $\left(\omega / \omega_{c}\right)$. Alternatively, this can be expressed as the summation

$$
1=\frac{\omega_{p}^{2}}{\omega_{c}^{2}} \sum_{n=0}^{\infty} \int_{-2 \pi(n+1)}^{-2 \pi n} \exp i \Omega \theta \sin \theta J_{0}\left(2 \mu \sin \frac{\theta}{2}\right) d \theta
$$

Substitution of $[\varphi-2 \pi(n+1)]$ for $\theta$ reduces the summation to

$$
1=\frac{\omega_{p}^{2}}{\omega_{c}^{2}} \sum_{n=0}^{\infty}(\exp [-i 2 \pi \Omega])^{n+1} \int_{0}^{2 \pi} \exp (i \Omega \varphi) \sin \varphi J_{0}\left(2 \mu \sin \frac{\varphi}{2}\right) d \varphi
$$

If the presence of a small loss component is assumed, this can be further simplified to

$$
\exp [i 2 \pi \Omega]-1=\frac{\omega_{p}^{2}}{\omega_{c}^{2}} \int_{0}^{2 \pi} \exp (i \Omega \varphi) \sin \varphi J_{0}\left(2 \mu \sin \frac{\varphi}{2}\right) d \varphi
$$

which separates into the two forms,

$$
\begin{aligned}
& \cos 2 \pi \Omega=1+\frac{\omega_{p}^{2}}{\omega_{c}^{2}} \int_{0}^{2 \pi} \cos \Omega \varphi \sin \varphi J_{o}\left(2 \mu \sin \frac{\varphi}{2}\right) d \varphi \\
& \sin 2 \pi \Omega=\frac{\omega_{p}^{2}}{\omega_{c}^{2}} \int_{0}^{2 \pi} \sin \Omega \varphi \sin \varphi J_{0}\left(2 \mu \sin \frac{\varphi}{2}\right) d \varphi
\end{aligned}
$$

either of which can be used for computation.

These can be regarded as general formulas from which integral expressions for velocity distributions other than the $\delta$-function can be deduced. As an example, for the Maxwellian of (24) we must evaluate the weighting integral

$$
\left(\frac{m}{2 \pi \kappa T_{e}}\right) \int_{0}^{\infty} J_{o}\left(2 \mu \sin \frac{\varphi}{2}\right) \exp \left(-\frac{m v_{R}^{2}}{2 \kappa T_{e}}\right) 2 \pi v_{R} d v_{R}
$$


The Bessel function identity required is [Bateman Manuscript Project, 1953]

$$
\int_{0}^{\infty} J_{0}\left(2 \mu \sin \frac{\varphi}{2}\right) \exp \left(-\frac{\mu^{2}}{2 \lambda}\right) \mu d \mu \equiv \lambda \exp \left(-2 \lambda \sin ^{2} \frac{\varphi}{2}\right)
$$

The dispersion relation is consequently given by either of the expressions

$$
\begin{aligned}
& \cos 2 \pi \Omega=1+\frac{\omega_{p}^{2}}{\omega_{c}^{2}} \exp (-\lambda) \int_{0}^{2 \pi} \cos \Omega \varphi \sin \varphi \exp (\lambda \cos \varphi) d \varphi \\
& \sin 2 \pi \Omega=\frac{\omega_{p}^{2}}{\omega_{c}^{2}} \exp (-\lambda) \int_{0}^{2 \pi} \sin \Omega \varphi \sin \varphi \exp (\lambda \cos \varphi) d \varphi
\end{aligned}
$$

The integral relations can be used to determine the series forms of (28) if appropriate Bessel function identities are used. For example, differentiation of (18) with respect to $\mu$ yields

$$
\sin \varphi J_{0}\left(2 \mu \sin \frac{\varphi}{2}\right) \equiv \sum_{n=1}^{\infty} \frac{2 n}{\mu} \frac{\partial}{\partial \mu}\left[J_{n}^{2}(\mu)\right] \sin n \theta,
$$

which can be substituted in (34) and integrated term by term. Similarly for the Maxwellian distribution we have [Bateman Manuscript Project, 1953]

$$
\exp (\lambda \cos \varphi) \equiv \sum_{n=0}^{\infty} \cos n \varphi I_{n}(\lambda)
$$

which can be differentiated and substituted in (37). Integration, term by term, then gives (28).

\section{References}

Bateman Manuscript Project (1953), Higher transcendental functions, 2, pp 7, 81, 102, and 50 (McGraw-Hill Book Co., Inc., New York, N.Y.).

Bazhanova, A. E., V. T. Karpukhin, A. N. Karkhov, and V. I. Pistunovich (1962), Cyclotron and thermal radiations of plasma in Ogra, Proceedings of the Conference on Plasma Physics and Controlled Nuclear Fusion Research, Salzburg, August 1961. Nucl. Fusion Suppl. 1, 227-232.

Bekefi, G., J. D. Coccoli, E. B. Hooper, and S. J. Buchsbaum (1962) Microwave emission and absorption at cyclotron harmonics of a warm plasma, Phys. Rev. Letters 9, No. 1, 6-9.

Bekefi, G., J. L. Hirshfield, and S. C. Brown (1961a), Kirchhoff's radiation law for plasmas with non-Maxwellian distributions, Phys. Fluids 4, No. 2, 173-176.

Bekefi, G., J. L. Hirshfield, and S. C. Brown (1961b), Cyclotron emission from plasmas with non-Maxwellian distributions, Phys. Rev. 122, No. 4, 1037-1042.

Bekefi, G., and E. B. Hooper (1964), Cyclotron radiation of an $\mathrm{Hg}$ plasma generated by an electron beam, Appl. Phys. Letters 4, No. 8, 135-136.

Bernstein, I. B. (1958), Waves in a plasma in a magnetic field, Phys. Rev. 109, No. 1, 10-21.

Bohm, D., and E. P. Gross (1949), Theory of plasma oscillations A: origin of medium-like behavior, Phys. Rev. 75, No. 12, 1851-1864.

Bohm, D., and E. P. Gross (1949), Theory of plasma oscillations B: Excitation and damping of oscillations, Phys. Rev. 79, No. 12, 992-1001.

Brown, S. C., and G. Bekefi (1962) Radio-frequency emission from plasmas not in thermodynamic equilibrium, Proc. Conf. on Plasma Physics and Controlled Nuclear Fusion Research, Salzburg, August, 1961. Nuclear Fusion Suppl. 3, 10891099.

Buchsbaum, S. J., and A. Hasegawa (1964), Excitation of longitudinal plasma oscillation near electron cyclotron harmonics, Phys. Rev. Letters 12, No. 25, 685-688.

Budden, K. G. (1961), Radio waves in the ionosphere (Cambridge Univ. Press, Cambridge)

Calvert, W., and G. B. Goe (1963), Plasma resonances in the upper ionosphere, J. Geophys Res. 68, No. 22, 6113-6120.

Canobbio, E., and R. Croci (1964), Harmonics of the electron cyclotron frequency in a PIG-discharge, Proc. 6th Intern. Conf. on Ionization Phenomena in Gases, Paris, July 1963 (SERMA Pub. Co., Paris) 3, 269-271.

Crawford, F. W. (1961) Electrostatic sound wave modes in a plasma, Phys. Rev. Letters 6, No. 12, $663-665$.

Crawford, F. W. (1963), The mechanism of Tonks-Dattner plasma resonances, Phys. Letters 5, No. 4, $244-247$.

Crawford, F. W. (1964a), Internal resonances of a discharge column, J. Appl. Phys. 35, No. 5, 1365-1369. 
Crawford, F. W. (1964b), A review of cyclotron harmonic phenomena in plasmas, Microwave Laboratory Report No. 1215, Stanford University.

Crawford, F. W. (1964c), Excitation of ion cyclotron harmonic waves in plasmas, Microwave Laboratory Report No. 1216, Stanford University.

Crawford, F. W., and G. S. Kino (1964), Cyclotron harmonic amplification in beams and plasmas, Microwave Laboratory Report No. 1204, Stanford University, Stanford, California.

Crawford, F. W., G. S. Kino, and A. B. Cannara (1963), Dipole resonances of a plasma in a magnetic field, J. Appl. Phys. 34, No. 11, 3168-3175.

Crawford, F. W., G. S. Kino, and H. H. Weiss (1964a), Excitation of cyclotron harmonic resonances in a mercury-vapor discharge, Phys. Rev. Letters 13, No. 7, 229-232.

Crawford, F. W., G. S. Kino, and H. H. Weiss (1964b), Transmission characteristics of cyclotron harmonic waves in plasma, Microwave Laboratory Report No. 1210, Stanford University, Stanford, Calif.

Crawford, F. W., and J. A. Tataronis (1965), Absolute instabilities of perpendicularly-propagating plasma waves, Microwave Laboratory Report No. 1295, Stanford University, Stanford, California.

Dreicer, H. (1964), Emission of cyclotron radiation from a PIG discharge, Bull. Am. Phys. Soc. 9, No. 3, 512.

Fejer, J. A., and W. Calvert (1964), Resonance effects of electrostatic oscillations in the ionosphere, J. Geophys. Res. 69, No. 23, 5049-5062.

Fields, H., G. Bekefi, and S. C. Brown (1961), Microwave measurements of the radiation temperature of a plasma in a magnetic field, Proc. 5th Intern. Conf. on Ionization Phenomena in Gases, Munich, Germany, August 1961 (North-Holland Pub. Co., Amsterdam) 1, 367-375.

Gross, E. P (1951), Plasma oscillations in a static magnetic field, Phys. Rev. 82, No. 2, 232-24,2.

Gruber, S., W. D. McBee, and L. T. Shepherd (1964) Observation of spatially growing waves at the cyclotron harmonics in an electron-beam-generated He plasma, Appl. Phys. Letters 4, No. 8, 137-138.

Harris, E. G. (1959), Unstable plasma oscillations in a magnetic field, Phys. Rev. Letters 2, No. 2, 34-36.

Harris, E. G. (1961), Plasma instabilities associated with anisotropic velocity distributions, J. Nucl. En., Pt. C, 2, No. 2, $138-145$.

Harp, R. S. (1965), Propagation of longitudinal plasma oscillations near cyclotron harmonics, Appl. Phys. Letters 6, No. 3, 51-53.

Hirshfield, J. L., and S. C. Brown (1961), Incoherent microwave radiation from a plasma in a magnetic field, Phys. Rev. 122, No. 3, 719-725.

Kubo, H., K. Mitani, S. Tanaka, and Y. Terumichi (1964), Microwave radiation from a plasma in a magnetic field (II), J. Phys. Soc., Japan 19, No. 2, 221-226.

Landau, L. (1946), On the vibrations of the electronic plasma, J. Phys. USSR 10, No. 1, 25-34.

Landauer, G. (1961), Magnetfeldabhängige Mikrowellenstrahlung aus einer He-Gasentladung, Proc. 5th Intern. Conf. on Ionization Phenomena in Gases, Munich, Germany, July 1961 (North-Holland Pub. Co., Amsterdam) 1, 389-394.

Landauer, G. (1962), Generation of harmonics of the electron-gyrofrequency in a Penning discharge, J. Nucl. Energy, Pt. C. 4, No. 6, 395-400.

Landauer, G. (1964), Experimental study on the emission of cyclotron harmonics, Bull. Am. Phys. Soc. 9, No. 3, 312.

Little, P. F. (1962) Ion waves in a bounded plasma, Nature, 194, 1137-1139.

Lockwood, G. E. K. (1963), Plasma and cyclotron spike phenomena observed in top-side ionograms, Can. J. Phys. 41, 190-194.

Lustig, C. W., W. D. McBee, and A. Kalisky (1964), Wide range microwave spectroscopy of noise radiation from magnetoplasmas, Rev. Sci. Instr. 35, No. 7, 869-871.

Malmberg, J. H., and C. B. Wharton (1964), Collisionless damping of electrostatic plasma waves, Phys. Rev. Letters 13, No. 6, 184-186.

Malmfors, K. G. (1950), Unstable oscillations in an electron gas, Arkiv Fysik 1, No. 30, 569-578.

Mitani, K., and H. Kubo (1961), On radiation from plasma in a static magnetic field, J. Phys. Soc., Japan, 16, $1480-1481$.

Mitani, K., H. Kubo, and S. Tanaka (1964a), Experiments on the negative radiation temperature at cyclotron resonance in plasma, Proc. 5th Intern. Conf. on Ionization Phenomena in Gases, Paris, July 1963 (SERMA Pub. Co., Paris) 3, 281285.

Mitani, K., H. Kubo, and S. Tanaka (1964b), Microwave radiation from a plasma in a magnetic field (I), J. Phys. Soc., Japan 19, No. 2, 211-220.

Nickel, J. C., J. V. Parker, and R. W. Gould (1963), Resonance oscillations in a hot nonuniform plasma column, Phys. Rev. Letters 11, No. 5, 183-185.

Ohara, S. (1962), Negative resistance due to Ramsauer-effect in plasma, Phys. Fluids 5, No. 11, $1483-1484$.

Ohara, S. (1963), Negative resistance in rare gases, J. Phys. Soc. (Japan) 18, No. 6, 852-863.

Parker, J. V., J. C. Nickel, and R. W. Gould (1964), Resonance oscillations in a hot nonuniform plasma, Phys. Fluids 7, No. 9, 1489-1500.

Pistunovich, V. I., and V. D. Shafranov (1961), Cyclotron radiation by ions in plasma, Nucl. Fusion 1, No. 3, $189-194$.

Ratcliffe, J. A. (1959), Magneto-ionic theory and its applications to the ionosphere (Cambridge Univ. Press, Cambridge).

Schneider, J. (1959), Stimulated emission of radiation by relativistic electrons in a magnetic field, Phys. Rev. Letters 2 , No. 12, 504-505.

Sen, H. K. (1952), Solar 'enhanced radiation' and plasma oscillations, Phys. Rev. 88, No. 4, 816-822. 
Stix, T. H. (1962), The Theory of Plasma Waves, lst ed., chs. 8, 9 (McGraw-Hill Book Co., Inc., New York).

Sturrock, P. A. (1965), Dipole resonances in a homogenous plasma in a magnetic field, Phys. Fluids 8, No. 1, 88-96.

Tanaka, S., and H. Kubo (1964), Microwave radiation at cyclotron harmonics in a positive column, Institute of Plasma Physics Report No. 23, Nagoya University, Nagoya, Japan.

Tanaka, S., K. Mitani, and H. Kubo (1963a), Microwave radiation from a plasma in a magnetic field, Institute of Plasma Physics Report No. 13, Nagoya University, Nagoya, Japan.

Tanaka, S., Y. Terumichi, K. Mitani, and H. Kubo (1963b), Anomalous microwave radiation at cyclotron resonance in partially ionized plasmas, J. Phys. Soc. Japan 18, No. 12, 1810-1821.

Twiss, R. Q. (1958), Radiation transfer and the possibility of negative absorption in radio astronomy, Australian J. Phys. 11, No. 4, 564-579.

Wharton, C. B. (1960), Microwave radiation measurements of very hot plasma, Proc. 4th Intern. Conf. on Ionization Phenomena in Gases, Uppsala, Sweden, August 1959 (North-Holland Pub. Co. Amsterdam) 2, 737-742.

(Paper 69D6-512) 